\title{
Scanning Electron Microscope 3D Surface Reconstruction via Optimization
}

Yasamin Sartipi ${ }^{1}$, Aidan Ross ${ }^{2}$, Weiwei Zhang ${ }^{3}$, Samuel Norris ${ }^{3}$, Hesham El-Sherif ${ }^{3}$, Christopher K. Anand ${ }^{1}$, Nabil D. Bassim ${ }^{3}$

${ }^{1}$ Department of Computing and Software, McMaster University, Hamilton, Ontario, Canada

${ }^{2}$ School of Computational Science and Engineering, McMaster University, Hamilton, Ontario, Canada

${ }^{3}$ Department of Materials Science and Engineering, McMaster University, Hamilton, Ontario, Canada

In this work, we present an optimization method for three-dimensional (3D) reconstruction of scanning electron microscope (SEM) images based on angle-of-incidence effects. Therefore, there would be less need to perform further time-consuming tests such as atomic force microscopy (AFM), so as to analyze the 3D topography. The proposed method requires a small number of images taken after rotating the stage. In this paper we illustrate the method using eight angles, unlike stereo-photogrammetry methods requiring hundreds of images [1]. At each angle we acquire a Backscatter Electron (BSE) and Secondary Electron (SE) image using an Everhart-Thornley detector. The pointwise sum of these images provides additional information about the slope of the surface.

Reconstructing the height is a two-step process. In the first step, we solve an optimization problem which can be thought of as finding the height map whose derivative in each of the directions for which we have opposing BSE image pairs most closely matches the differences. Previous methods have integrated along one direction, either directly or via an optimization problem, making them susceptible to parallel line artefacts [2]. In the second step, we solve an optimization problem which finds a new height map which most closely matches the gradient vector field of the first height map, scaled so that the slope at each pixel matches the expected slope based on the combined SE image. We use an empirical model to map SE image intensity to slope.

We produced a number of test objects fabricated using focused ion beam (FIB) patterning, collected both SEM images and Atomic Force Microscopy (AFM) data for comparison. Figure 1 shows three of our test objects fabricated in a silicon substrate. For each we show one BSE and one SE source image, the AFM height map represented as an image and a 3D rendering, as well as the BSE-only height map represented as an image and a 3D rendering. We did not apply the second step to these examples, because we have not calibrated the SE intensity for silicon. Both the AFM probe and our method have limitations which make it hard to quantify the accuracy of our algorithm, but we are satisfied with them qualitatively. For example, the AFM probe (with thickness $10 \mathrm{~nm}$ ) was not able to track the sharp angles in the Fireball, whereas it reported the depths of the trenches in the third test object to be close to equal, whereas our method computed shallower depths for the narrower trenches.

The second innovation involves the combination of SE-mode information with the difference of BSEmode images. Figure 2 shows the results for a single tin ball in which the top BSE-only results are clearly not rotationally symmetric (left) and not very round (right), whereas the bottom BSE+SE results are visibly rotationally symmetric, and much rounder.

\section{References}

[1] J Lebiedzik, Scanning 2 (1979), p. 230-237.

[2] AP Tafti et al, Micron 78 (2015), p. 54-66. 

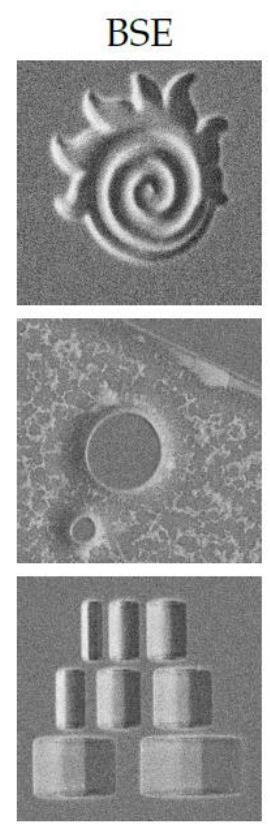
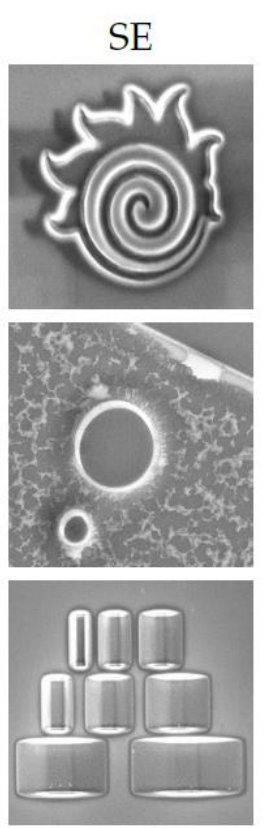
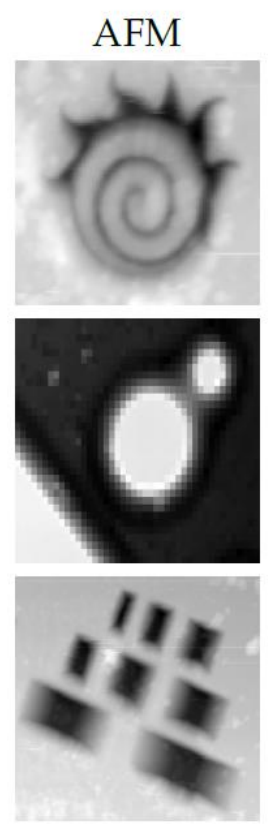
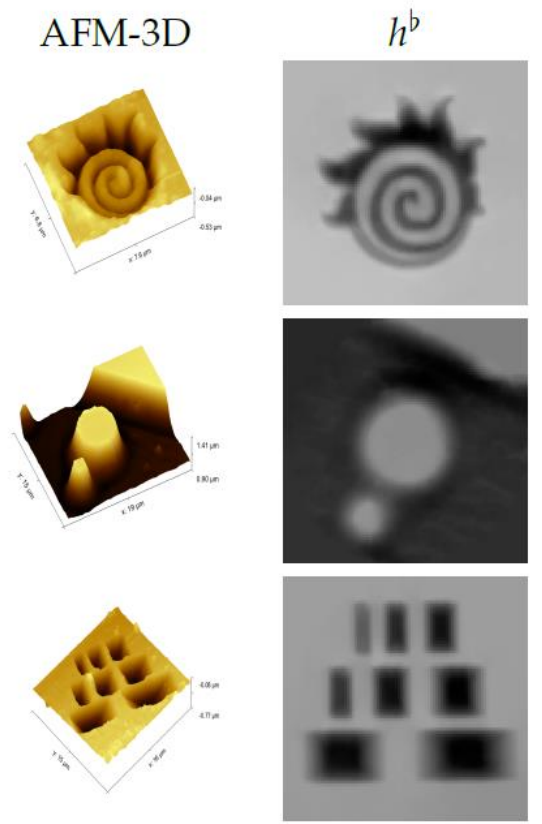

$h^{b}-3 \mathrm{D}$
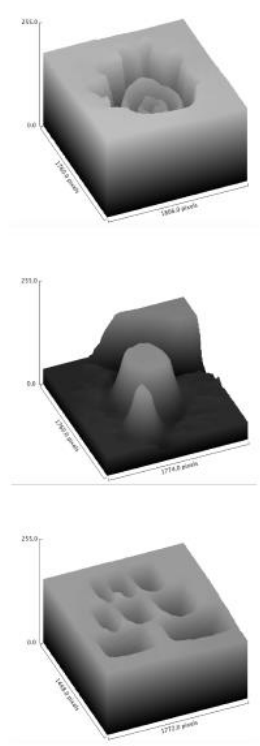

Figure 1. Validation of the method on silicon test objects: McMaster Fireball, towers and trenches. The columns show sample BSE and SE images, the AFM height map, a 3D rendering of the AFM height map, the optimized height map and a $3 \mathrm{D}$ rendering thereof.
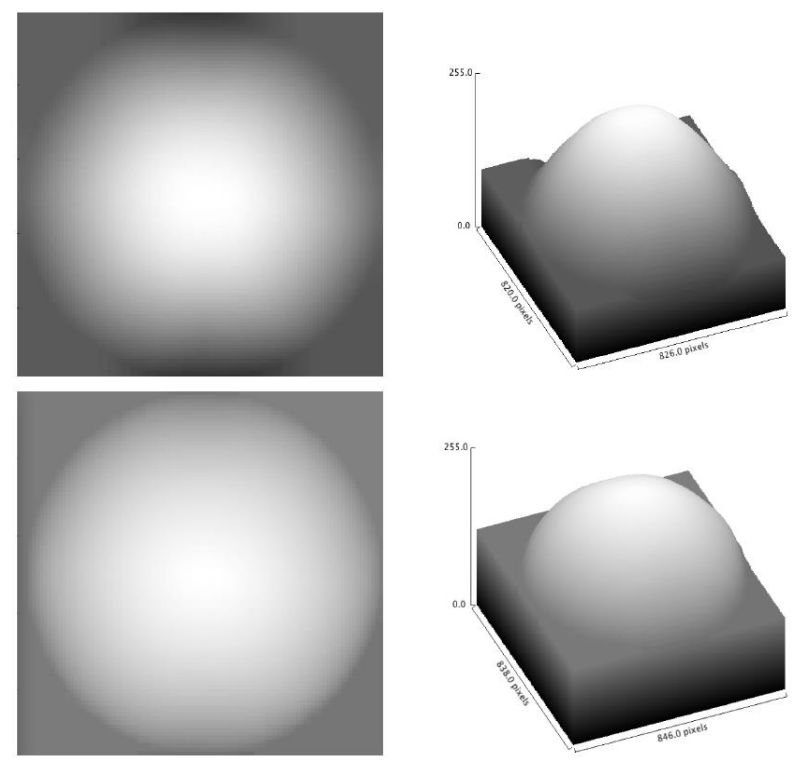

Figure 2. SE information produces rotationally symmetric and rounder ball. Top: two views of the height map produced by the first step. Bottom: two views of the height map produced by BSE+SE which uses the direction of first step height map and the SE images 